\title{
Ultrafast Charge Separation at a Polymer-Single-Walled Carbon Nanotube Molecular Junction
}

\author{
Samuel D. Stranks, Christian Weisspfennig, Patrick Parkinson, Michael B. Johnston, \\ Laura M. Herz, and Robin J. Nicholas*
}

Department of Physics, Clarendon Laboratory, Parks Road, Oxford, OX1 3PU, U.K.

\begin{abstract}
We have investigated the charge photogeneration dynamics at the interface formed between single-walled carbon nanotubes (SWNTs) and poly(3-hexylthiophene) (P3HT) using a combination of femtosecond spectroscopic techniques. We demonstrate that photoexcitation of P3HT forming a single molecular layer around a SWNT leads to an ultrafast ( 430 fs) charge transfer between the materials. The addition of excess P3HT leads to long-term charge separation in which free polarons remain separated at room temperature. Our results suggest that SWNT-P3HT blends incorporating only small fractions (1 \%) of SWNTs allow photon-to-charge conversion with efficiencies comparable to those for conventional (60:40) P3HT-fullerene blends, provided that small-diameter tubes are individually embedded in the P3HT matrix.
\end{abstract}

KEYWORDS Carbon nanotube, P3HT, charge separation, molecular junction

$\mathrm{O}$ rganic photovoltaic (OPV) cells have been demonstrated as low-cost alternatives to silicon-based cells, offering the possibility of low temperature "reel-to-reel" fabrication, necessary for viable large-scale power generation. ${ }^{1}$ The majority of OPV devices utilize exciton dissociation at the interface between two materials which exhibit a type-II heterojunction alignment, ${ }^{2}$ resulting in interfacial charge separation and free polarons in the materials. However, it has been shown that for many OPV materials such as polymer-polymer blends, exciton dissociation leads to the formation of an interfacial bound geminate charge pair, termed an exciplex. ${ }^{3-7}$ This species can be strongly bound and long-lived, significantly lowering charge separation efficiencies.

Single-walled carbon nanotubes (SWNTs) are promising candidates for use in OPVs because of their large aspect ratios, high carrier mobilities and their controllable solubilization in polymer solution. ${ }^{8-10}$ Despite this, devices based on SWNT blends with conjugated polymers have to date shown poor performance. ${ }^{1-14}$ However, these results may be explained by recent experimental ${ }^{15}$ and theoretical ${ }^{16}$ studies demonstrating that a type-II heterojunction only exists for certain interfaces, such as between small diameter semiconducting SWNTs and regioregular poly(3-hexylthiophene) (P3HT). Even for such blends, energy transfer from the polymer to the SWNTs may compete effectively against charge transfer.

* To whom correspondence should be addressed. E-mail: r.nicholas1@ physics.ox.ac.uk.

Received for review: 08/5/2010

Published on Web: 11/24/2010
In this letter, we show that charge transfer across the interface between individually dispersed SWNTs wrapped in a monolayer sheath of P3HT occurs on an ultrafast (430 fs) time scale. Generated charge pairs relax into a bound interfacial charge-transfer state or via nonradiative recombination of excitons within the nanotubes and no long-lived free polarons are observed. However, in the presence of an excess P3HT network, charge separation at room temperature is long-lived and comparable to that in a conventional P3HT-fullerene blend. This is the first time such blends have been studied using femtosecond time resolution, and we observe a charge transfer time three-magnitudes faster than reported previously. ${ }^{17,18}$ In addition, significant long-term charge separation is observed for the first time in SWNTpolymer blends. We conclude that charge separation is only possible if small-diameter semiconducting tubes are individually embedded in an excess P3HT matrix. Our findings explain the poor polymer-SWNT device behavior to date and provide a promising route to incorporation of SWNTpolymer blends into OPVs.

In recent work, Schuettfort et al. ${ }^{19}$ synthesized a highly purified nanohybrid structure consisting of a SWNT coated with a monolayer of P3HT. In these purified nanohybrids, the P3HT organizes into a sheathlike morphology on the tubes, aligned at an approximate angle of $48^{\circ}$ with respect to the SWNT axis ${ }^{20}$ and at a distance of twice the van der Waals radius $(0.34 \mathrm{~nm})$ from the nanotube; ${ }^{19}$ this is shown schematically in Figure 1b. In addition, a small number of isolated P3HT nanocrystals of typical dimensions of $3-5 \mathrm{~nm}$ are observed attached to the P3HT sheath enveloping the tubes, as shown by atomic force microscopy (AFM) (Figure $1 \mathrm{C},{ }^{19}$ ) and transmission electron microscopy $(\mathrm{TEM})^{11}$ im- 

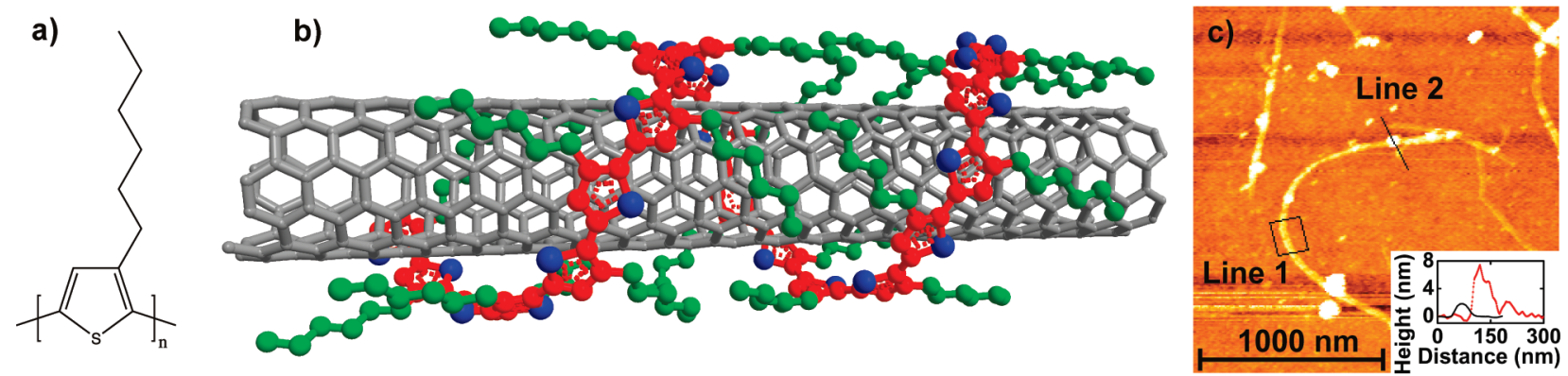

FIGURE 1. (a) Chemical structure of P3HT. (b) Schematic diagram of a $(6,5)$ SWNT coated with a monolayer of sheath P3HT. Sulfur atoms are colored blue, the carbon backbone is red and the side-chains are green. (c) AFM image showing the isolated nanotubes coated with P3HT sheath and the scattered P3HT nanocrystals. Inset: Heights of AFM slices marked on image, where Line 1 is the black solid line and Line 2 is the broken red line. The heights are consistent with nanotubes of diameter $0.7-0.9 \mathrm{~nm}$ coated with a monolayer of P3HT and with nanocrystals of typical dimensions $3-5 \mathrm{~nm}$ on the sheath.

ages. These morphologies will be referred to as P3HT sheath and P3HT nanocrystals, respectively.

Five thin-film samples were studied: (1) a bulk P3HT control, (2) P3HT-NT nanohybrids as described above prepared using the methods described by Schuettfort et al., ${ }^{19}$ and (3) a sample referred to in the following as P3HT-NT(1 \% ) consisting of an overall concentration of $1 \%$ SWNTs wrapped in a P3HT sheath surrounded by excess P3HT, or network P3HT. Finally, sample (4), a conventional 60:40 blend of P3HT-[6,6]-phenyl- $\mathrm{C}_{61}$-butyric acid methyl ester (PCBM) and sample (5) a film of SWNTs embedded in an inert gelatin matrix were prepared to allow comparative studies. Further details of sample preparation and all measurement techniques employed are given in the Supporting Information.

Absorption measurements (Perkin-Elmer Lambda 9 UVvis-NIR Spectrophotometer) of the films are presented in Figure 2. For the P3HT-containing samples, the spectral region between $350-650 \mathrm{~nm}$ is dominated by P3HT absorption of similar magnitude. As expected, the nanotube $\mathrm{E}_{11}$ transitions in the region 950-1350 nm do not appear in the bulk P3HT control and the nanotube $\mathrm{E}_{22}$ transitions are clearly seen in the NT-gelatin control $(400-800 \mathrm{~nm})$. A photoluminescence excitation (PLE) map for the P3HT-NT film samples is also presented in Supporting Information. Together, these measurements demonstrate that $(6,5)$ semiconducting nanotubes are the dominant species in the films. The manufacturers (SouthWest NanoTechnologies) specify that less than $10 \%$ of the nanotubes are metallic.

Figure 3 shows the steady-state photoluminescence (PL) spectra for the different samples excited at $400 \mathrm{~nm}$. Significant NT emission (950-1100 nm) is only observed from the P3HT-NT sample and exhibits a large red shift relative to the empirical values deduced by Weisman et al. ${ }^{21}$ This red shift is a direct result of the type-II heterojunction alignment of the materials as predicted by Kanai and Grossman ${ }^{16}$ and observed by Schuettfort et al. ${ }^{15}$ The observed NT emission probably results from the transfer of excitations from P3HT to the NTs as $400 \mathrm{~nm}$ photons are predominantly absorbed by the P3HT and none of the van Hove singularities of the nanotubes present fall into this region.

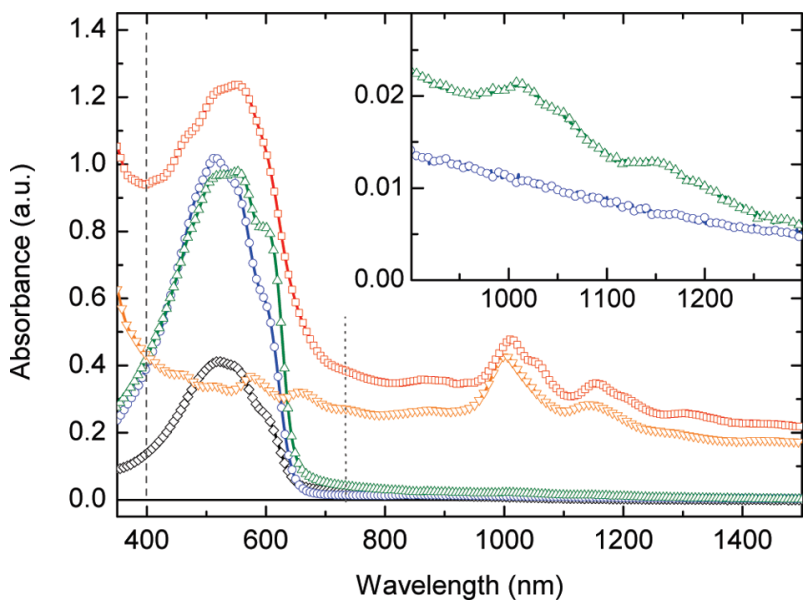

FIGURE 2. Absorption spectra for P3HT (blue circles), P3HT-NT (red squares), P3HT-NT(1\%) (green triangles) and P3HT-PCBM (black diamonds) samples as spun-cast on quartz, along with that for the gelatin-NT sample (orange inverted triangles). The gray dashed vertical line shows the excitation wavelength used for time-resolved measurements $(400 \mathrm{~nm})$, while the gray dotted vertical line shows the probe wavelength for transient absorption measurements (730 $\mathrm{nm})$. Inset: Region clearly showing the $\mathrm{E}_{11}$ nanotube peaks in the P3HT-NT(1\%) sample.

All three P3HT-containing samples show emission associated with P3HT (Figure 3, 600-900 nm) but with very different intensities and spectral shapes. The pure P3HT control sample shows a PL spectrum typical for aggregated P3HT, whose lamellar structure permits efficient electronic coupling between parallel chains leading to a red shift in the emission $^{22,23}$ and the suppression of the lowest (0-0) peak in the Franck-Condon series associated with the $\mathrm{C}-\mathrm{C}$ stretch vibration. ${ }^{24-27}$ Such suppression has been shown to result fundamentally from the high symmetry of the ensemble state comprising a number of identical chains over which the excitation becomes delocalized. ${ }^{26}$ In contrast, the P3HT emission from the P3HT-NT sample shows a clear 0-0 emission peak and a blue shift with respect to the bulk P3HT emission. This suggests that for the P3HT-NT sample, emission originates from the P3HT sheath, which comprises a polymer monolayer whose $\pi-\pi$ interactions are with the NT only, and therefore is not subject to emission quenching 


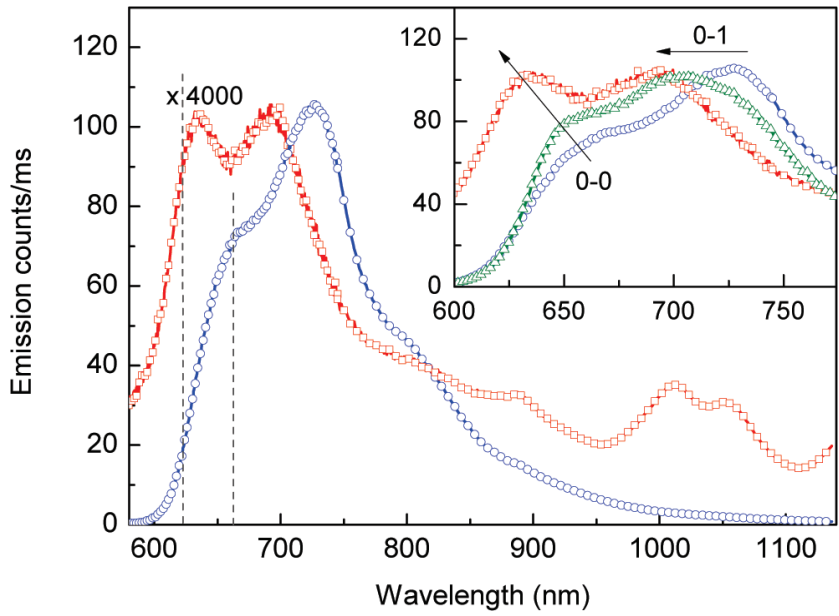

FIGURE 3. Steady-state PL spectra for P3HT (blue circles) and P3HT-NT (red squares, $\times 4000$ ) samples. Data were taken with an InGaAs detector above $745 \mathrm{~nm}$ and a Si CCD below and carefully scaled to match and allow comparison. The gray dashed lines indicate the detection wavelength used for the TCSPC and upconversion measurements for each sample, namely the 0-0 P3HT transition wavelength in the bulk P3HT $(660 \mathrm{~nm})$ and P3HT-NT (620 $\mathrm{nm})$ samples. Inset: Magnified view of the P3HT emission from the samples, including that from the P3HT-NT(1\%) sample (green triangles, $\times 40$ ).

arising from such symmetry constraints. Additional P3HT nanocrystalline aggregates present in the P3HT-NT samples (Figure 1) do not appear to contribute to the emission, probably because the PL quantum efficiency for the chainlike P3HT sheath is $10 \%$ as opposed to $<1 \%$ for aggregates ${ }^{28-30}$ and their small size and proximity to the NT will also cause some PL quenching. For the P3HT-NT(1\%) sample, the P3HT emission has a shape between that for the P3HT-NT and the bulk P3HT samples but resembling more closely the latter, suggesting a dominant contribution from aggregated network P3HT

Figure 3 also shows that in the presence of nanotubes significant quenching of the sheath P3HT emission is observed. The intensity differences of the emission are striking; the spectrum from bulk P3HT is of the order of 4000 times more intense than from P3HT-NT and 40 times more intense than for the P3HT-NT(1\%). These observations suggest that the intimate contact between sheath P3HT and the nanotubes leads to highly efficient quenching of the excitations placed on the P3HT.

To determine the speed and mechanism for the quenching of photoexcitations on the P3HT we conducted both time-resolved PL and transient absorption measurements with subpicosecond time resolution. As detailed below, the former allowed us to determine the dynamics of photoexcitation quenching on P3HT while the latter yielded information about the time-scales and extent of the free charge generation.

Time-resolved photoluminescence of the P3HT in the samples was measured using two techniques: time-correlated single photon counting (TCSPC) with time resolution

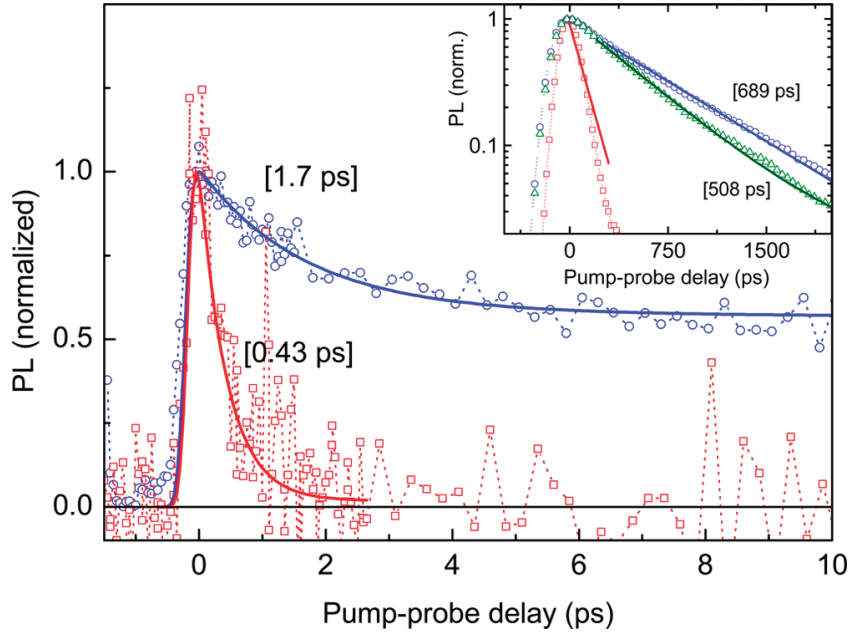

FIGURE 4. Normalized PLUC results for the samples following 400 $\mathrm{nm}$ sample excitation $\left(0.15 \mu \mathrm{J} / \mathrm{cm}^{2}\right.$ fluence). The emission from the P3HT sample (blue circles) was detected at $660 \mathrm{~nm}$ and that for the P3HT-NT (red squares) was detected at $620 \mathrm{~nm}$ where detection wavelengths correspond to the P3HT 0-0 transition in each sample. Similar dynamics are also observed for the bulk sample when excited at $620 \mathrm{~nm}$. Appropriate fits give fast time constants of 0.43 and 1.7 ps for P3HT-NT and P3HT samples, respectively, including a slow component for the P3HT sample only. Inset: TCSPC decay curves at the same probe wavelengths used for PLUC following excitation at $400 \mathrm{~nm}\left(9 \mathrm{~nJ} / \mathrm{cm}^{2}\right.$ fluence). The P3HT-NT(1\%) (green triangles) sample was also detected at $660 \mathrm{~nm}$.

of $\sim 120$ ps and photoluminescence up-conversion (PLUC) with resolution of $220 \mathrm{fs}$. Measurements were taken at the peaks corresponding to the steady-state P3HT 0-0 transition in each sample, that is, $620 \mathrm{~nm}$ for the P3HT-NT sample and $660 \mathrm{~nm}$ for the pure P3HT and P3HT-NT(1 \% ) samples. Figure 4 shows the time-resolved PL decay measured for bulk P3HT and P3HT-NT samples using the high-resolution PLUC technique (the P3HT-NT(1\%) PLUC data exhibit a very similar behavior to the bulk P3HT sample over the displayed time window and hence are not shown). Lowresolution TCSPC data (inset) for the bulk P3HT and P3HTNT(1\%) samples display a faster PL decay for the latter with monoexponential fits yielding lifetimes of 689 and 508 ps, respectively. However, the TCSPC technique is unable to time-resolve the PL from the P3HT-NT sample sufficiently since the measured decay ( $\tau \sim 117 \mathrm{ps}$ ) is identical to the system response function. On the other hand, the PLUC data reveal ultrafast PL decay components for the three samples. To extract time constants for these ultrafast components, the PL decay for the P3HT-NT sample was fitted with a single exponential, and that for the P3HT sample with the sum of two exponentials with one of the two time-constants set to that of the observed long-term decay (689 ps). These functions were convoluted with a Gaussian system response function of width 220 fs with best fits obtained shown in Figure 4 as solid lines.

For bulk P3HT, a fast initial component with time constant of 1.7 ps is extracted, which is likely to be caused by initial excitation relaxation processes mediated, for example, 
by vibrational or torsional cooling and migration through a density of states associated with energetic disorder. ${ }^{27,31-33}$ Following such relaxation, the emission from bulk P3HT is generally long-lived, as would be expected. In contrast, the sheath emission in the P3HT-NT sample decays completely to zero with an ultrafast time constant of 0.43 ps. This extremely rapid decay of the P3HT emission from P3HT-NT films is consistent with the factor 4000 quenching of its timeintegrated emission relative to that of the bulk P3HT (Figure 3), as discussed previously.

Our observations can be compared with previous results by Geng et al. ${ }^{17}$ and Chen et al. ${ }^{18}$ who studied P3HT and poly(9,9-dioctylfluorenyl-2,7-diyl) (PFO) wrapped SWNTs, respectively, using TCSPC to conclude that energy transfer occurred on a time scale of order 400-500 ps. However, these studies were based on films that had a large amount of excess free polymer dominating the emission. The TCSPC data for the film containing excess P3HT, P3HT-NT(1\%), shows a decay of the residual P3HT emission of 508 ps that is only slightly faster than the bulk P3HT (689 ps), probably due to regions of excess polymer far from an interface with a NT, in agreement with these previous studies. However, as we demonstrate below, the low resolution of TCSPC masks the fast exciton quenching processes occurring directly at the polymer-NT heterointerface. Therefore, only the use of the PLUC technique has allowed us to observe the true polymer exciton quenching in the presence of NTs, which is three orders-of-magnitude faster than suggested by the previous studies.

To determine whether the P3HT emission quenching in the blend samples is caused by charge separation at the interface, we conducted ultrafast transient absorption measurements on the samples. The presence of free charges was monitored as a function of time after excitation by measuring the pump-induced changes in transmission of a probe at a wavelength of $730 \mathrm{~nm}$, the peak of the absorption for the P3HT delocalized polaron. ${ }^{34}$ The exciting pump pulse was at a photon wavelength of $400 \mathrm{~nm}$ and had a fluence of $50 \mu \mathrm{J} / \mathrm{cm}^{2}$ at the focus.

Figure 5 displays the transient absorption $(-\Delta \mathrm{T} / \mathrm{T})$ data obtained for the four P3HT-containing samples. P3HT-PCBM blends are known for their significant photon-to-charge conversion ratios, ${ }^{35}$ and the expected well-known trend for the P3HT-PCBM (60:40) control sample is observed with a long-lived absorption arising from charge separated polaron pairs. ${ }^{36,37}$ The transient response from the P3HT bulk sample is also consistent with previously reported observations showing an initial decreased absorption that has been attributed to stimulated emission from excitons originating from some regions of regiorandom P3HT. ${ }^{28,36,37}$ Excitonexciton annihilation processes, which are significant with the fluences used here, ${ }^{38}$ cause a fast decay of the absorption signal, which may also contain a very small transient component arising from charge absorption originating from internal hot exciton dissociation intrinsic to the polymer. ${ }^{39-41}$

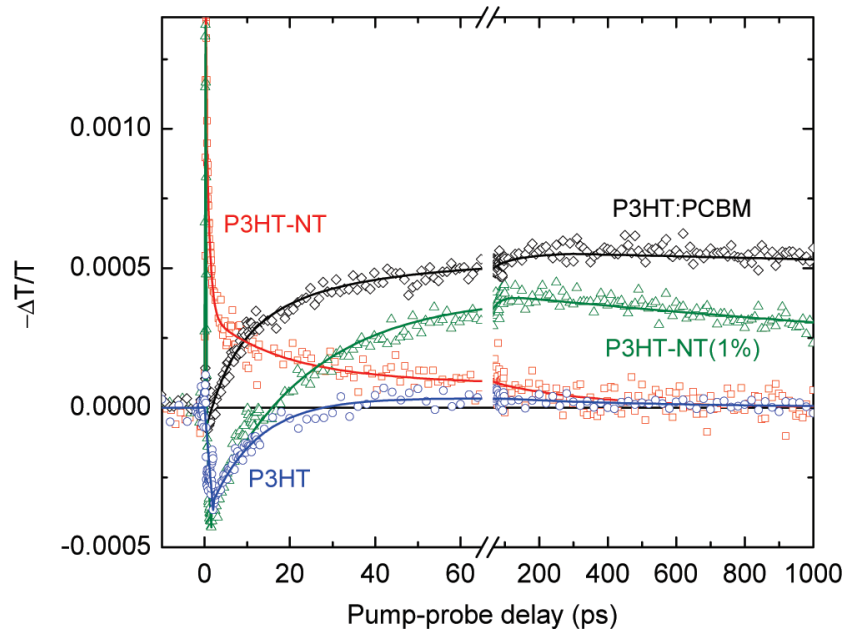

FIGURE 5. Relative transmission changes for varying pump-probe delays for the P3HT-PCBM (black diamonds), P3HT (blue circles), P3HT-NT (red squares), and P3HT-NT(1 \%) (green triangles) samples when excited at $400 \mathrm{~nm}$ and probed at $730 \mathrm{~nm}$. A pump fluence of $50 \mu \mathrm{J} / \mathrm{cm}^{2}$ was used. Solid lines are a guide to the eye.

As expected, no long-term charge-separated state exists for the bulk P3HT sample.

The P3HT-NT signal shows no negative stimulated emission signal but rather an increased absorption that, in contrast to P3HT-PCBM, decays relatively quickly to zero. The absence of the stimulated emission signal is expected given that PLUC data show the quenching of sheath P3HT excitons on an ultrafast time scale of $400 \mathrm{fs}$. In addition, the P3HT nanocrystals attached to some of the NTs (Figure 1) are highly regioregular with no regiorandom domains, and thus very low stimulated emission rates should be expected. ${ }^{28,36,37}$ The strong absorption signal has an ultrafast ( $\sim 350 \mathrm{fs})$ rise and a decay that occurs on several time scales. These data suggest that fast quenching of the P3HT emission in P3HT - NT films is accompanied by a rise in the presence of free charges on the P3HT. However, these free charges are clearly not long-lived with the majority of the charge absorption disappearing within 10 ps after excitation and most of the rest decaying within another $\sim 100 \mathrm{ps}$. The free holes must therefore be rapidly transferred to states with low quantum efficiency, to account for the strong (4000x) PL quenching. These could either be the nanotube excitons, which are known to have strong Auger recombination at high excitation intensities ${ }^{42-45}$ occurring on a time scale of $1-5$ ps or bound charge-transfer complexes formed across the interface, similar to exciplexes, which are known to have low radiative efficiencies. ${ }^{3,4,7}$ Figure 3 shows that although weak in absolute terms, the nanotube PL becomes relatively more significant due to its shorter exciton lifetimes $(\sim 100$ $\mathrm{ps}^{42,43,46}$ ) whereas no exciplex emission, which should occur in a similar wavelength region, is obviously visible. The NT emission is expected to dominate since exciplex lifetimes are known to be of order nanoseconds and above. ${ }^{3,4,7}$ Nevertheless, such exciplex-like states have been shown to play a significant role in a variety of polymer-containing 
blends with type-II heterointerfaces ${ }^{3-7}$ and are thus likely to be also of importance here.

However, the P3HT-NT(1\%) sample containing excess P3HT shows significantly different behavior. During the first 10 ps the data reveal enhanced transmission arising from stimulated emission caused by the majority of carriers which are excited in the network P3HT (including some regiorandom) regions. The subsequent dynamics are similar to those for the P3HT-PCBM sample: a rapid growth in the free polaron absorption indicates an effective charge separation process with a rise time of 20-30 ps reflecting the migration time of excitons to the heterointerface. The peak value of the charge absorption signal is also similar to that observed for the P3HT-PCBM sample. Accounting for differences in sample absorption at the excitation wavelength, we estimate that the peak charge generation efficiency in the P3HT$\mathrm{NT}(1 \%)$ sample is $40 \%$ of that found for the standard P3HT-PCBM blend with an optimized mass ratio. ${ }^{36}$ The fact that the quenched PL data show a dominant contribution from aggregated network P3HT indicates there are occasional large regions of purely bulk P3HT in the P3HT-NT(1 \%) sample. The small fraction of P3HT excitons formed in these domains are unable to reach a P3HT-NT interface before recombining and hence do not contribute to free charge generation. Therefore, further optimization of both the film morphology and the P3HT-NT blend ratio may result in an even higher charge-generation being obtained. Removal of any remaining metallic nanotubes $(<10 \%)$, which are liable to lead to energy transfer, would also improve the charge generation. The free-charge absorption for the P3HT-NT(1 \%) sample also shows a slight decline over the time window (1 ns) investigated. However, an accurate determination of the charge lifetime in these systems will require measurements over microsecond time scales, for example, as those described in ref 6.

To understand the dynamic processes occurring at the polymer-NT heterointerface, we consider the schematic shown in Figure 6. For an exciton excited within the polymer, charge transfer at an interface will result in the electron being clearly confined to the nanotube. The energy offset controlling the hole behavior is, however, marginal. As a result, although the hole is most likely to reside on the polymer side of the interface, it probably also has a significant probability for activated transfer to the NT. For the sheath morphology, the hole located on the polymer is geometrically constrained to be in close proximity to the nanotube and the formation of a bound state charge-transfer compelex (or "exciplex") is therefore a likely scenario. However, such a charge-transfer state at the interface will differ from those typically observed for polymer-polymer heterojunctions, where it exists across two strands with similar dimensions in which fairly localized particles have similar effective masses. For the NT-polymer interface, in contrast, the electron on the nanotube is delocalized around the tube and has a mass for motion along the tube of only

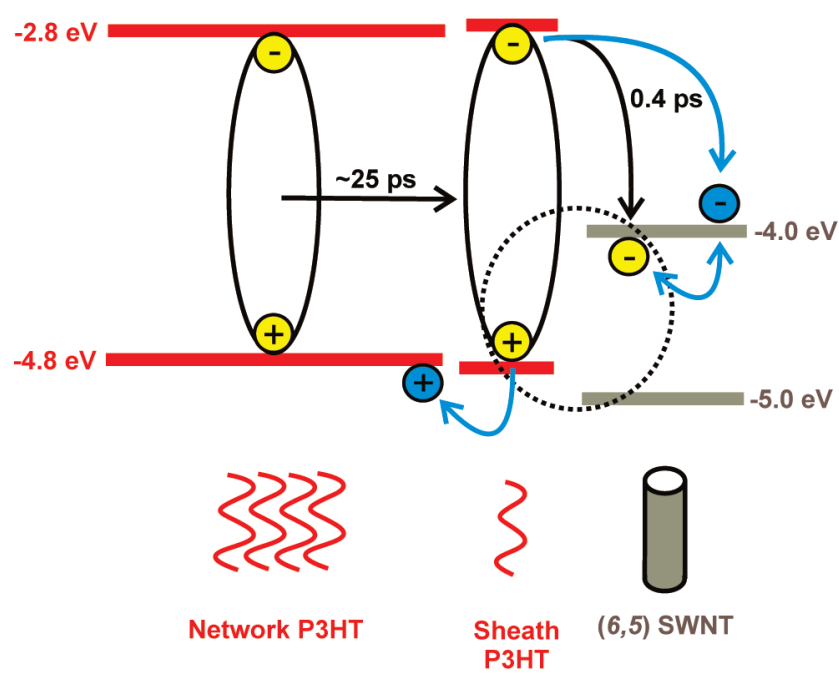

FIGURE 6. Scheme to illustrate the observed charge generation dynamics for a P3HT sheath monolayer around a $(6,5)$ SWNT with an excess of P3HT acting as a surrounding network. A bound exciton is created on the network P3HT and migrates to a sheath-P3HT-NT interface within $\sim 25$ ps. At the interface the exciton dissociates and an electron is transferred to the nanotube while the hole remains on the P3HT. This produces free polarons (blue). In the presence of the network P3HT, the hole can migrate away from the interface (blue) and immediate charge separation is achieved. In the absence of network P3HT, the free polarons form a weakly bound exciplex or recombine nonradiatively as excitons within the nanotubes (both processes represented by dotted line). Vacuum energy level values are as described in refs 19 and 47 with a slightly larger bandgap for sheath P3HT than network P3HT in line with observed absorption (Figure 2) and PL (Figure 3) data.

one-tenth of the free electron mass. ${ }^{48}$ There is therefore a large mismatch in both wave function spread and particle masses for an exciplex composed of a charge pair across a nanotube-polymer interface. We estimate conservatively that this will reduce the binding energy of any exciplex by a factor of order $4^{49}$ compared to polymer-polymer heterojunction analogues ${ }^{3}$ where binding energies are typically 100-200 meV. In summary, examination of the band structures leads to the conclusion that at the NT-polymer interface, the generated charge pairs will experience weak Coulomb interactions and comprise an electron wave function that is delocalized along the NT and a hole wave function that is mainly located on the polymer but may cross over into the NT. The charge pairs may therefore decay either through recombination of the charges across the interface, or while they are both located on the NT.

The addition of network polymer, as in the P3HT-NT(1\%) sample, will significantly alter this picture by allowing the hole to migrate away from the interface thus eluding such recombination processes. In addition, the expanded dielectric environment of the complex may subtly alter the energetic level alignment at the interface making stable charge separation more favorable and further decreasing the exciplex binding energy. As a result, efficient long-lived charge separation is expected once additional P3HT is added to the individually polymer-wrapped nanotubes. 
In the P3HT-NT sample, the PLUC data show that the exciton which is created on the sheath P3HT is quenched on a time scale of $0.4 \mathrm{ps}$, and transient absorption traces indicate that free polarons are produced within this time. These then decay away on a time scale of order 10-100 ps. We attribute these observations to the ultrafast charge dissociation of P3HT excitons at the heterointerface. However, because the nanotubes are wrapped by individual P3HT chains, the positive charge on the P3HT cannot move away from the interface and the generated charge pair will decay through recombination of the charges either across the interface, or while they are both located on the NT.

When the network P3HT is added, the majority of excitons are generated initially in the network P3HT. Transient absorption data show that free charges form in P3HT on a time scale of $20-30$ ps (signal rise time), as the excitons move to an interface between a sheath-P3HT layer and a NT. This value is consistent with the quenching of the timeintegrated polymer PL intensity by a factor of 40 relative to the bulk P3HT sample. At the interface, rapid dissociation of the exciton into free polarons again occurs but the majority of free holes generated are now long-lived. This is consistent with the picture that holes on the P3HT are now able to migrate away from the P3HT-NT interface thereby eluding capture. Therefore, the excess P3HT network provides a medium allowing extraction of the free holes and effective charge separation, in accordance with the predictions by Schuettfort et al. ${ }^{15}$

It is emphasized that the scheme presented in Figure 6 is only valid for small diameter nanotubes that establish a defined type-II alignment with P3HT and for the case of welldispersed nanotubes. For larger diameter tubes, the alignment becomes type-I and, as observed experimentally, ${ }^{19}$ energy transfer becomes the dominant process. Lioudakis et al. ${ }^{50}$ also conducted transient absorption studies on polymer-NT films, but examined larger diameter HiPCO nanotubes dispersed by P3HT. Consequently, their data do not show any evidence of charge separation even with the same polymer-SWNT ratio as that used in the work presented here. Our study has shown, therefore, that significantly improved photovoltaic devices need to utilize suspensions of these purified, small diameter nanohybrids incorporated at small volume fraction in a polymer matrix. Devices to date have yet to satisfy both conditions since all have so far incorporated larger diameter HiPCO, arcdischarge or chemical vapor deposition tubes. ${ }^{11-14}$

In conclusion, we have investigated the charge photogeneration dynamics at the interface formed between smalldiameter semiconducting SWNTs and a monolayer coating of P3HT. ${ }^{19}$ Despite the poor performance shown by devices to date, such blend materials are promising candidates for use in OPVs owing to their type-II heterojunction alignment, ${ }^{15,16}$ high mobilities, and large aspect ratios. ${ }^{8,51}$ We have demonstrated that photoexcitation of the sheath P3HT leads to an ultrafast electron transfer to the nanotube on a time scale of $400 \mathrm{fs}$. Since the remaining hole on the P3HT is confined to this single molecular layer, either a bound charge-transfer complex forms across the interface or nonradiative recombination occurs on the nanotube and no generation of free charge occurs. However, the introduction of an excess of P3HT surrounding the molecular complexes allows transport of the hole away from the interface, leading to long-term charge separation. Our results suggest that small diameter SWNT-P3HT nanohybrids act as efficient charge generation interfaces for use in photovoltaic devices, provided that they are embedded in a matrix with a sufficient excess of P3HT. The nanotube-polymer interfaces can provide comparable or better dissociation interfaces than all-polymer junctions. In addition, nanotubes offer greater electron mobility and longer percolation paths than conjugated polymers, suggesting that polymer-nanotube composites may be more effective active media in OPVs than polymer-polymer or polymer-fullerene blends. Significantly, we have demonstrated that the presence of only $1 \%$ nanotubes is able to produce a similar efficiency of charge separation to that observed in an optimized 60:40 blend of P3HT-fullerene for which the fullerene electron acceptor does not contribute to light absorption. These findings thus establish a promising route for developing efficient OPV devices utilizing polymer-SWNT blends.

Acknowledgment. The authors thank the Engineering and Physical Sciences Research Council for their financial support, H. Swain and J. Alexander-Webber for taking the AFM images, T. Schuettfort for useful discussions and N. Saranrom for making the gelatin samples. S. Stranks acknowledges the Rhodes Trust for financial support.

Supporting Information Available. Preparation methods for blend films of P3HT with SWNT or PCBM, and SWNTgelatin films. Description of apparatus used for photoluminescence and transient absorption measurements. This material is available free of charge via the Internet at http:// pubs.acs.org.

\section{REFERENCES AND NOTES}

(1) Snaith, H. J.; Schmidt-Mende, L. Adv. Mater. 2007, 19, $3187-$ 3200 .

(2) Tang, C. W. Appl. Phys. Lett. 1986, 48, 183

(3) Morteani, A. C.; Sreearunothai, P.; Herz, L. M.; Friend, R. H.; Silva, C. Phys. Rev. Lett. 2004, 92, 247402

(4) Offermans, T.; Meskers, S. C. J.; Koetse, M. M.; Janssen, R. A. J. Phys. Rev. B 2005, 72, No. 045213.

(5) Yin, C.; Kietzke, T.; Neher, D.; Horhold, H. H. Appl. Phys. Lett. 2007, 90, No. 092117

(6) Ohkita, H.; Cook, S.; Astuti, Y.; Duffy, W.; Tierney, S.; Zhang, W.; Heeney, M.; McCulloch, L.; Nelson, J.; Bradley, D. D. C.; Durrant, J. R. J. Am. Chem. Soc. 2008, 130, 3030

(7) Benson-Smith, J. J.; Wilson, J.; Dyer-smith, C.; Mouri, K.; Yamaguchi, S.; Murata, H.; Nelson, J. J. Phys. Chem. B 2009, 113, $7794-$ 7799.

(8) Reich, S.; Thomsen, C.; Maultzsch, J. Carbon Nanotubes - Basic Concepts and Physical Properties, 1st ed.; Wiley-VCH: New York, 2004.

(9) Nish, A.; Hwang, J. Y.; Doig, J.; Nicholas, R. J. Nat. Nanotechnol. 2007, 2, 640-646 
(10) Hwang, J. Y.; Nish, A.; Doig, J.; Douven, S.; Chen, C. W.; Chen, L. C.; Nicholas, R. J. J. Am. Chem. Soc. 2008, 130, 3543-3553.

(11) Geng, J. X.; Zeng, T. Y.J. Am. Chem. Soc. 2006, 128, 16827-16833.

(12) Kymakis, E.; Servati, P.; Tzanetakis, P.; Koudoumas, E.; Kornilios, N.; Rompogiannakis, I.; Franghiadakis, Y.; Amaratunga, G. A. J. Nanotechnology 2007, 18, 435702 .

(13) Mallajosyula, A. T.; Iyer, S. S. K.; Mazhari, B. J. Appl. Phys. 2010 108, 094902

(14) Arranz-Andres, J.; Blau, W. J. Carbon 2008, 46, 2067-2075.

(15) Schuettfort, T.; Nish, A.; Nicholas, R. J. Nano Lett. 2009, 9, 3871 3876

(16) Kanai, Y.; Grossman, J. C. Nano Lett. 2008, 8, 908-912.

(17) Geng, J. X.; Kong, B. S.; Yang, S. B.; Youn, S. C.; Park, S.; Joo, T.; Jung, H. T. Adv. Funct. Mater. 2008, 18, 2659-2665.

(18) Chen, F. M.; Zhang, W. J.; Jia, M. L.; Wei, L.; Fan, X. F.; Kuo, J. L.; Chen, Y.; Chan-park, M. B.; Xia, A. D.; Li, L. J. J. Phys. Chem. C 2009, 113, 14946-14952

(19) Schuettfort, T.; Snaith, H.; Nish, A.; Nicholas, R. Nanotechnology 2010, 21, No. 025201

(20) Goh, R. G. S.; Motta, N.; Bell, J. M.; Waclawik, E. R. Appl. Phys. Lett. 2006, 88, No. 053101

(21) Weisman, R. B.; Bachilo, S. M. Nano Lett. 2003, 3, 1235-1238.

(22) Gutierrez-llorente, A.; Horowitz, G.; Perez-Casero, R.; Perriere, J.; Fave, J. L.; Yassar, A.; Sant, C. Org. Electron. 2004, 5, 29-34.

(23) Jiang, X. M.; Österbacka, R.; An, C. P.; Vardeny, Z. V. Synth. Met. 2003, 137, 1465-1468.

(24) Spano, F. C. Chem. Phys. 2006, 325, 22-35

(25) Clark, J.; Silva, C.; Friend, R. H.; Spano, F. C. Phys. Rev. Lett. 2007 98, 206406

(26) Spano, F. C.; Clark, J.; Silva, C.; Friend, R. H.J. Chem. Phys. 2009, 130, No. 074904

(27) Parkinson, P.; Müller, C.; Stingelin, N.; Johnston, M. B.; Herz, L. M. J. Phys. Chem. Lett. 2010, 1, 2788-2792.

(28) Korovyanko, O. J.; Österbacka, R.; Jiang, X. M.; Vardeny, Z. V.; Janssen, R. A. J. Phys. Rev. B 2001, 64, 235122

(29) Sheng, C. X.; Tong, M.; Singh, S.; Vardeny, Z. V. Phys. Rev. B 2007, 75, No. 085206

(30) Theander, M.; Inganäs, O.; Mammo, W.; Olinga, T.; Svensson, M.; Andersson, M. R. J. Phys. Chem. B 1999, 103, 7771-7780

(31) Westenhoff, S.; Beenken, W. J. D.; Friend, R. H.; Greenham, N. C.; Yartsev, A.; Sundström, V. Phys. Rev. Lett. 2006, 97, 166804
(32) Trotzky, S. J. Phys. D: Appl. Phys. 2009, 42, No. 055105.

(33) Chang, M. H.; Hoffmann, M.; Anderson, H. L.; Herz, L. M. J. Am. Chem. Soc. 2008, 130, 10171-10178.

(34) Österbacka, R.; An, C. P.; Jiang, X. M.; Vardeny, Z. V. Science 2000, 287, 839-842

(35) Savenije, T. J.; Kroeze, J. E.; Yang, X. N.; Loos, J. Adv. Funct. Mater. $2005,15,1260-1266$

(36) Hwang, I. W.; Moses, D.; Heeger, A. J.J. Phys. Chem. C 2008, 112, 4350

(37) Piris, J.; Dykstra, T. E.; Bakulin, A. A.; Knulst, W.; Trinh, M. T.; Schins, J. M.; Siebbeles, L. D. A. J. Phys. Chem. C 2009, 113, 14500-14506

(38) Guo, J. M.; Ohkita, H.; Benten, H.; Ito, S. J. Am. Chem. Soc. 2009, $131,16869-16880$.

(39) Dicker, G.; Siebbeles, L. D. A.; Warman, J. M. Phys. Rev. B 2004, 70, No. 045203

(40) Ai, X.; Beard, M. C.; Knutsen, K. P.; Shaheen, S. E.; Rumbles, G.; Ellingson, R. J. J. Phys. Chem. B 2006, 110, 25462-25471.

(41) Hendry, E.; Koeberg, M.; Schins, J. M.; Siebbeles, L. D. A.; Bonn, M. Chem. Phys. Lett. 2006, 432, 441

(42) Xu, X.; Chuang, K.; Nicholas, R. J.; Johnston, M. B.; Herz, L. M. J. Phys. Chem. C 2009, 113, 18106-18109.

(43) Huang, L. B.; Krauss, T. D. Phys. Rev. Lett. 2006, 96, No. 057407

(44) Wang, F.; Dukovic, G.; Knoesel, E.; Brus, L. E.; Heinz, T. F. Phys. Rev. B 2004, 70, 241403.

(45) Ma, Y. Z.; Valkunas, L.; Dexheimer, S. L.; Bachilo, S. M.; Fleming, G. R. Phys. Rev. Lett. 2005, 94, 157402

(46) Hagen, A.; Steiner, M.; Raschke, M. B.; Lienau, C.; Hertel, T.; Qian, H. H.; Meixner, A. J.; Hartschuh, A. Phys. Rev. Lett. 2005, 95, 197401

(47) Chua, L. L.; Zaumseil, J.; Chang, J. F.; Ou, E. C. W.; Ho, P. K. H.; Sirringhaus, H.; Friend, R. H. Nature 2005, 434, 194-199.

(48) Pedersen, T. G. Phys. Rev. B 2003, 67, No. 073401.

(49) Perebeinos, V.; Tersoff, J.; Avouris, P. Phys. Rev. Lett. 2004, 92, 257402

(50) Lioudakis, E.; Othonos, A.; Alexandrou, I. Nanoscale Res. Lett. 2008, 3, 278-283

(51) Sirringhaus, H.; Tessler, N.; Friend, R. H. Science 1998, 280, 1741 